\title{
Coste-efectividad de la atención del paciente con diabetes mellitus tipo 2 manejado por el médico familiar versus estrategia educativa
}

\author{
Enrique Villarreal-Ríosa , Emma Rosa Vargas-Dazaa, Lidia Martínez-Gonzáleza , \\ Liliana Galicia-Rodríguez ${ }^{a}$ e Isabel Ramos-Arteaga ${ }^{a, b}$.
}

\begin{abstract}
a Unidad de Investigación Epidemiológica y en Servicios de Salud de Querétaro. Instituto Mexicano del Seguro Social. Querétaro (Querétaro). México.

b Unidad de Medicina Familiar $\mathrm{n}^{\circ}$ 16. Instituto Mexicano del Seguro Social. Querétaro (Querétaro). México.
\end{abstract}

Correspondencia: Enrique Villarreal Ríos. Avda. 5 de febrero, s/n, esq. Avda. Ignacio Zaragoza, Colonia Centro. C.P. 76000 - Querétaro (Querétaro de Arteaga). México. Correo electrónico: felibree@infosel. net.mx

Recibido el 28 de mayo de 2013.

Aceptado para su publicación el 17 de julio de 2013.

\section{RESUMEN}

Objetivo: Evaluar el coste-efectividad de la atención del paciente con diabetes mellitus tipo 2 manejado por el médico familiar versus estrategia educativa.

Diseño del estudio: Estudio coste-efectividad.

Participantes: Pacientes con diabetes mellitus tipo 2 integrados en dos grupos: pacientes atendidos por médico familiar y pacientes insertos en una estrategia educativa.

Mediciones principales: La efectividad evaluada con la modificación de los valores bioquímicos: glucemia, colesterol total y triglicéridos. El coste estimado en dólares americanos (\$US) definido por la intensidad de uso y el coste unitario de los servicios de medicina familiar, laboratorio, medicamentos y la estrategia educativa. El análisis incluyó relación coste-efectividad y proyección.

Resultados: La mejor relación coste-efectividad para la glucosa plasmática se presenta en la alternativa estrategia educativa, por cada 100 \$US invertidos la glucosa disminuye 10,42 mg/ dl; en la estrategia médico familiar por la misma cantidad desciende $2,57 \mathrm{mg} / \mathrm{dl}$. La tendencia es similar para el colesterol y los triglicéridos; el índice de masa corporal no presentó descenso en ninguna de las alternativas.

Conclusiones: La mejor relación coste-efectividad se identifica en la estrategia educativa.

Palabras clave: Diabetes Mellitus, Educación, Medicina Familiar y Comunitaria, Análisis CosteBeneficio.

\section{ABSTRACT}

Cost effectiveness of type 2 Diabetes Mellitus patient care handled by family doctor versus educational strategy.

Objective: To assess the cost effectiveness of type 2 Diabetes Mellitus patient care handled by family doctor versus educational strategy.

Design: Cost effectiveness study.

Participants: Type 2 Diabetes Mellitus patients falling into two groups: patients under family doctor and those in educational strategy.

Main Surveyed Data: The assessed effectiveness with modification of the biochemical values: glycemia, total cholesterol and triglycerides. The estimated cost in US Dollars is defined by rate of use and unit cost for family medicine services, laboratory, medicines and educational strategy. The analysis included cost effectiveness ratio and projection.

Results: The best cost effectiveness ratio for plasma glucose is presented in the educational strategy alternative; for every 100 US $\$$ spent, glucose is reduced $10,42 \mathrm{mg} / \mathrm{dl}$. In family doctor strategy, the reduction is $2,57 \mathrm{mg} / \mathrm{dl}$ for the same amount. The trend is similar for cholesterol and triglycerides; the body mass index showed no reduction in either of the alternatives.

Conclusions: The best cost effectiveness ratio is found in educational strategy.

Key words: Diabetes Mellitus, Education, Family and Community Medicine, Cost-Benefit analysis. 


\section{INTRODUCCIÓN}

El aumento de pacientes con diabetes mellitus tipo $2^{1}$ y sus complicaciones ${ }^{2,3}$ representa un reto para las instituciones responsables de su atención al asignarse entre dos y tres veces los recursos destinados a la salud ${ }^{4}$, por lo que ante la limitación de recursos se plantea la necesidad del establecimiento de estrategias eficientes de atención y manejo del paciente con estas características.

En la actualidad las estrategias de atención incluyen la consulta del médico familiar y la atención multidisciplinaria en la cual la educación a la salud se convierte en el eje de acción ${ }^{5,6}$.

En la atención de medicina familiar el paciente con diabetes mellitus tipo 2 (DM2) es citado mensualmente $^{7}$, no obstante pareciera ser que no se ha logrado el control de esta población. En la literatura se ha señalado que el uso frecuente de los servicios de salud no implica una vía de control $^{8}$, al respecto existen reportes que refieren que aproximadamente el $70 \%$ de los pacientes manejan niveles de glucosa superiores a los establecidos como meta ${ }^{9,10}$.

En las estrategias educativas se combinan las acciones de consulta personalizada y sesiones educativas que se desarrollan en un lapso de 12 meses con la finalidad de que el paciente integre y desarrolle habilidades de autocuidado en el manejo y control de este problema crónico degenerativo ${ }^{11}$. Los reportes en torno a los pacientes que pertenecen a grupos educativos refieren que aproximadamente el $58 \%$ manejan niveles de glucosa en sangre considerados como normales ${ }^{12}$.

En el contexto de la economía de la salud la elección de una modalidad para implantarla como política pública requiere de la evaluación económica, específicamente el análisis coste-efectividad, que permite examinar los costes y consecuencias de las estrategias de manera conjunta ${ }^{13-15}$ y establecer cuál tiene la mejor relación coste-efectividad para la institución dada la limitación de recursos para la atención de población usuaria.

En este contexto el objetivo del artículo es evaluar el coste-efectividad de la atención del paciente con diabetes mellitus tipo 2 manejado por el médico familiar versus estrategia educativa.

\section{SUJETOS Y MÉTODOS}

Se realizó un estudio de coste-efectividad en pacientes con diabetes mellitus tipo 2 usuarios de la Unidad de Medicina Familiar N ${ }^{\circ} 16$ del Instituto Mexicano del Seguro Social en la Ciudad de Querétaro durante el periodo de marzo de 2010 a febrero de 2011.
Se conformaron dos grupos de estudio: el primero integrado por pacientes insertos en una estrategia educativa y el segundo atendidos por un médico familiar. Para la integración de los grupos se realizó una invitación personal a cada paciente que acudió a la consulta con el médico familiar; aquellos que aceptaron la invitación se integraron al grupo de la intervención educativa; los que la rechazaron conformaron el grupo de atención en medicina familiar.

- Estrategia médico familiar: Atención mensual del médico familiar en el consultorio, con una duración de 15 minutos para valorar condiciones clínicas y bioquímicas del paciente.

- Estrategia educativa: La integración al grupo se da en tres dimensiones: la evaluación por el médico familiar y la enfermera para conocer los antecedentes, las condiciones clínicas, las condiciones bioquímicas y establecer la meta de control; la evaluación por el psicólogo y trabajadora social con fines motivacionales; y la integración nutricional realizada por la nutricionista. Mensualmente el paciente acude a una sesión educativa de dos horas y media de duración, con participación del médico familiar, enfermera, trabajadora social, nutricionista y familiares. El seguimiento del paciente se realiza durante un año y en cada visita, además de la educación, se evalúan los parámetros clínicos y bioquímicos. Al final del año el paciente regresa y continúa en control con el médico familiar.

Se incluyeron pacientes de ocho años o menos de evolución, con al menos tres glucosas registradas en un año de atención y que en la medición basal reportaron cifras de glucosa en sangre superiores a $130 \mathrm{mg} / \mathrm{dl}$. Se consideró como rango normal una glucosa en ayunas de 70 a $130 \mathrm{mg} / \mathrm{dl}^{14}$. Se excluyeron aquellos que recibieron atención fuera de la institución y los que presentaron retinopatía e insuficiencia renal crónica.

El tamaño de la muestra se calculó con la fórmula de porcentajes para dos poblaciones, con un nivel de confianza del $95 \%(\mathrm{Za}=1,64$, una cola), un poder de la prueba del $80 \%(Z b=0,84$, una cola), considerando que la efectividad en el grupo atendido por el médico familiar fue del $30 \%$ y la efectividad en el grupo de la estrategia educativa del $55 \%$. El total de la muestra fue de 45 por grupo; no obstante, se incrementó al doble y se trabajó con una relación de 2:1 para un total de 172 en el grupo de estrategia educativa y de 79 en el grupo de médico familiar.

La técnica muestral fue no aleatoria por cuota empleando como marco muestral para el grupo de médico familiar el listado nominal de pacientes diabéticos y para el grupo de estrategia educativa el listado nominal de los pacientes inscritos. 
Se estudiaron variables sociodemográficas (edad, sexo, estado civil, escolaridad y ocupación) y de salud (tiempo de diagnóstico, presión arterial sistólica y presión arterial diastólica).

- Efectividad: la efectividad se midió a corto plazo, utilizando como indicadores bioquímicos la glucosa venosa en ayunas, el colesterol total y los triglicéridos en ayunas; de ellos se obtuvo el promedio de las tres últimas mediciones del año, cifra que se contrastó con el valor basal y se cuantificó. La diferencia se empleó para evaluar la efectividad.

- Coste promedio estimado en dólares estadounidenses (\$US): el coste promedio se calculó multiplicando la intensidad de uso por el coste unitario de medicina familiar, laboratorio, farmacia, estomatología, nutrición, psicología, estrategia educativa y medicamentos.

- Intensidad de uso: la intensidad de uso de los servicios se estimó para un año y se identificó a partir del registro de consultas en medicina familiar, estudios de laboratorio, farmacia, estomatología, nutrición, psicología, estrategia educativa y medicamentos existentes en el expediente clínico, además del registro de asistencias al grupo de estrategia educativa.

- Coste unitario: coste unitario en medicina familiar, laboratorio, farmacia, estomatología, nutrición y psicología. Se identificaron los insumos necesarios para generar una atención (personal, mobiliario, equipo, instrumental, infraestructura, luz, teléfono, agua). Se investigó el gasto anual de cada uno de los insumos, con información obtenida en el departamento contable, administrativo y de personal de la institución de salud. El gasto anual por insumo se dividió entre el total de día laborables al año, y este resultado entre el total de minutos laborables al día. Posteriormente se determinó el total de minutos invertidos en generar una atención en medicina familiar y laboratorio. La multiplicación del coste del minuto por el total de minutos necesarios para generar una atención permitió identificar el coste por insumo por atención. La suma de los costes por insumo por atención generó el coste unitario de la atención en el servicio de medicina familiar y de laboratorio.

- Coste unitario de medicamentos: el coste unitario por envase de medicamento se obtuvo de los registros de compras consolidadas existentes en el departamento de administración de la institución de salud. Se identificó el número de unidades por envase por tipo de medicamento. La división del coste unitario por envase de medicamento entre el número de unidades por envase de medicamento se convirtió en el coste unitario por unidad de medicamento.
- Coste unitario de la estrategia educativa: el coste unitario de la estrategia educativa se estimó a partir de la identificación de los insumos utilizados (personal de salud, equipo, mobiliario, infraestructura y material didáctico). En los departamentos contable, de administración y de personal se identificó el gasto anual de cada insumo. Se identificó el total de sesiones al año. La división por tipo de insumo del gasto anual entre el número de sesiones permitió identificar el coste unitario por sesión. La suma del coste unitario por insumo permitió conocer el coste unitario de una sesión educativa.

- Coste-efectividad: el coste-efectividad se identificó para cada uno de los indicadores bioquímicos y antropométricos considerando la disminución del indicador y el coste. Se identificó el coste de la intervención y el total de $\mathrm{mg} / \mathrm{dl}$ modificados en cada intervención. Se calculó el coste por $\mathrm{mg} / \mathrm{dl}$ dividiendo el coste de la intervención entre el total de $\mathrm{mg} /$ dl disminuidos. Se calculó el total de $\mathrm{mg} / \mathrm{dl}$ modificados por cada 100 \$US.

- Proyecciones: proyección del coste por indicador. Para cada grupo se tomó como referencia la medición del indicador bioquímico $(\mathrm{mg} / \mathrm{dl})$. Se relacionó con el total de unidades modificadas por cada 100 \$US invertidos. Se proyectó para cada alternativa el nivel de mg/ dl por cada 100 \$US invertidos.

El análisis se realizó desde la perspectiva institucional y de la población para un año e incluyó porcentajes, promedios, análisis coste-efectividad y proyección.

\section{RESULTADOS}

\section{Características sociodemográficas}

En los grupos estudiados, estrategia educativa y médico familiar, la edad promedio fue de 54,14 años (IC95\%: 52,07 - 56,21) y 53,22 años (IC95\%: $50,58-55,86)$ respectivamente. Tanto en el grupo de estrategia educativa como en el grupo seguido por el médico familiar predominaron: el sexo femenino (68,9\%; IC95\%: $61,0-76,8)$ y $(64,6 \%$; IC95\%: $54,1-75,1)$, el estado civil casado $(71,2 \%$; IC95\%: 63,5 - 78,9) y (77,5\%; IC95\%: 68,3 - 86,7), la escolaridad primaria o menos (45,1\%; IC95\%: 36,6 - 53,6) y (42,6\%; IC95\%: 31,7 - 53,5) y la ocupación principal fue ama de casa $(53,8 \%$; IC95\%: 45,3 $62,3)$ y $(50,0 \%$; IC95\%: $39,0-61,0)$.

\section{Características de salud}

En el grupo estrategia educativa y en el grupo médico familiar la presión arterial sistólica fue de $124,47 \mathrm{mmHg}$ (IC95\%: 122,22 - 126,72) y 125,00 $\mathrm{mmHg}$ (IC95\%: 122,85 - 127,15), en tanto que la diastólica fue de $80,23 \mathrm{mmHg}$ (IC95\%: 79,33 
- 81,13) y 78,68 mmHg (IC95\%: 77,78 - 79,58) respectivamente.

\section{Efectividad}

La glucosa inicial en el grupo de estrategia educativa fue de $190,20 \mathrm{mg} / \mathrm{dl}$, y en el grupo atendido por médico familiar de $194,57 \mathrm{mg} / \mathrm{dl} \quad(p=0,61)$; los valores finales en cada grupo fueron $161,24 \mathrm{mg} /$ dl y $187,97 \mathrm{mg} / \mathrm{dl}(p=0,00)$ respectivamente. En el primer grupo el descenso fue de $28,96 \mathrm{mg} / \mathrm{dl}$ $(p=0,00)$ y en el segundo de $6,60 \mathrm{mg} / \mathrm{dl}(p=0,18)$. En la tabla 1 se presentan los valores iniciales, finales y la diferencia para cada uno de los indicadores evaluados.

\section{Coste}

El coste promedio en la estrategia educativa fue de 277,91 \$US y en el médico familiar de 256,60 \$US. El insumo más costoso en ambos casos corresponde a la consulta realizada. En la tabla 2 se presentan el uso, el coste unitario y el coste promedio por insumo y estrategia.

\section{Coste-efectividad}

Previo al análisis coste-efectividad se aseguró que en la medición basal ambos grupos, médico familiar y estrategia educativa, fueran similares en tiempo de evolución (4,30 años versus 4,00 años; $\mathrm{p}=0,33)$ y nivel de glucosa $(194,57 \mathrm{mg} / \mathrm{dl}$ versus $190,20 \mathrm{mg} / \mathrm{dl} ; \mathrm{p}=0,61)$.

En la estrategia educativa el promedio de glucosa disminuido fue de $28,96 \mathrm{mg} / \mathrm{dl}$ y en la atención otorgada por médico familiar de $6,59 \mathrm{mg} / \mathrm{dl}$. El coste por mg descendido fue de 9,60 \$US en la primera alternativa y 38,88 \$US en la segunda alternativa. Por cada 100 \$US invertidos se desciende 10,42 $\mathrm{mg} / \mathrm{dl}$ en la alternativa estrategia educativa y 2,57 $\mathrm{mg} / \mathrm{dl}$ en la estrategia médico familiar. En la tabla 3 se presenta esta misma información para el colesterol, triglicéridos e índice de masa corporal (IMC).

\section{Proyección}

La inversión de 400 \$US logra un nivel de glucosa de $119,56 \mathrm{mg} / \mathrm{dl}$ en la estrategia educativa y de $177,68 \mathrm{mg} / \mathrm{dl}$ en la estrategia médico familiar. En la tabla 4 se presenta el nivel de glucosa en relación a la inversión.

Cuando se invierten 600 \$US en la estrategia educativa el nivel de colesterol es de 190,46 mg/dl y de $197,86 \mathrm{mg} / \mathrm{dl}$ en la estrategia médico familiar, no obstante en ambas modalidades el incremento de la inversión no modifica mucho el nivel de colesterol en sangre. En la tabla 5 se presenta el valor de colesterol en sangre según el nivel de inversión en cada tipo de estrategia.

El nivel de triglicéridos no se modifica sustancialmente en la estrategia médico familiar cuando se incrementa la inversión. Cuando se invierten 300 \$US el nivel de triglicéridos en esta estrategia es $229,39 \mathrm{mg} / \mathrm{dl}$ y en la estrategia educativa es $200,21 \mathrm{mg} / \mathrm{dl}$. En la tabla 6 se presentan los niveles de triglicéridos por estrategia y nivel de inversión.

\section{DISCUSIÓN}

Para el manejo del paciente con diabetes mellitus los sistemas de salud han implementado estrategias de atención $n^{4}$. En el estudio que aquí se muestra, ubicado en el contexto de la economía de la salud, se presenta el resultado de la evaluación coste-efectividad de dos alternativas de atención institucional, resultados útiles en el proceso de toma de decisiones para la correcta asignación de recursos.

Es una realidad que en las investigaciones realizadas fuera del laboratorio el investigador no tiene el control total de la población y en consecuencia el control que debería existir sobre las variables de estudio se pierde. Ante este escenario existen dos posibilidades: o se renuncia al estudio o se asumen una serie de supuestos que de no cumplirse pueden influir en el resultado pero de los que nunca se tendrá la certeza. Este es el caso de la investigación que aquí se presenta y de toda la investigación que se realiza en grupos de población no controlados en laboratorio, no obstante se deberá reconocer que una buena parte de la investigación realizada y publicada se enfrenta ante este escenario. Unido a ello también se deberá reconocer que la no aleatorización, al limitar la posibilidad de los sujetos a formar parte de la muestra, se puede acompañar de sesgos que modifiquen el resultado, que en el caso del artículo que se presenta se refiere a conductas que pueden influir en el control de la glucosa en sangre.

En este estudio las estrategias de atención propuestas incluyen, además de la atención del médico familiar, estrategias educativas basadas en equipos multidisciplinarios que apuestan por la educación del paciente en las medidas de prevención y limitación del daño ${ }^{16}$. Y, aunque las estrategias educativas y la atención del médico familiar tienen diferentes procesos e insumos ${ }^{17}$, el objetivo final es el mismo, el control bioquímico y clínico del paciente diabético. En este escenario la glucosa, el colesterol, los triglicéridos y el IMC son parámetros a evaluar ${ }^{18,19}$.

En este artículo la evaluación estadística inicial de los parámetros bioquímicos y el tiempo de evolución identifican dos grupos homogéneos y por lo tanto susceptibles de compararse, y se puede interpretar como una fortaleza del trabajo que se complementa con la medida de efectividad empleada (diferencia de la medición inicial con la final). No obstante, se deberá reconocer que la diferencia inicial en el IMC es una debilidad; al respecto se debe comentar que el IMC, contra todo lo esperado, en los dos grupos 


\begin{tabular}{|c|c|c|c|c|c|c|}
\hline \multirow[t]{2}{*}{ Indicador } & \multicolumn{3}{|c|}{ Estrategia Educativa } & \multicolumn{3}{|c|}{ Médico Familiar } \\
\hline & Inicial & Final & Diferencia & Inicial & Final & Diferencia \\
\hline Glucosa (mg/dl) & 190,20 & 161,24 & 28,96 & 194,57 & 187,97 & 6,60 \\
\hline Colesterol (mg/dl) & 199,49 & 196,63 & 2,86 & 197,96 & 197,93 & 0,03 \\
\hline Triglicéridos (mg/dl) & 237,71 & 215,03 & 22,68 & 231,90 & 230,56 & 1,34 \\
\hline Índice de Masa Corporal $\left(\mathrm{kg} / \mathrm{m}^{2}\right)$ & 30,46 & 33,69 & $-3,23$ & 36,94 & 37,12 & $-0,18$ \\
\hline
\end{tabular}

Tabla 1. Modificación de los indicadores bioquímicos y antropométricos en el grupo de estrategia educativa y de médico familiar.

\begin{tabular}{|c|c|c|c|c|c|}
\hline \multirow{2}{*}{ Insumo } & \multicolumn{2}{|c|}{ Uso } & \multirow{2}{*}{$\begin{array}{l}\text { Coste } \\
\text { Unitario }\end{array}$} & \multicolumn{2}{|c|}{ Coste Promedio } \\
\hline & $\begin{array}{l}\text { Estrategia } \\
\text { Educativa }\end{array}$ & $\begin{array}{l}\text { Médico } \\
\text { Familiar }\end{array}$ & & $\begin{array}{l}\text { Estrategia } \\
\text { Educativa }\end{array}$ & $\begin{array}{l}\text { Médico } \\
\text { Familiar }\end{array}$ \\
\hline Charlas & 5,07 & 2,66 & 5,47 & 27,73 & 14,55 \\
\hline Laboratorio & & & 8,77 & 37,88 & 32,24 \\
\hline Glucosa & 5,28 & 4,45 & 6,00 & 31,68 & 26,70 \\
\hline Colesterol y triglicéridos & 2,24 & 2,00 & 2,77 & 6,20 & 5,54 \\
\hline Consulta Médico Familiar & - & 8,96 & 19,00 & - & 170,24 \\
\hline Consulta Estrategia Educativa & 7,86 & - & 11,50 & 90,39 & - \\
\hline Farmacia & 7,86 & 8,96 & 4,25 & 33,41 & 38,08 \\
\hline Estomatología & 4,90 & 0,03 & 18,06 & 88,49 & 0,54 \\
\hline Nutrición & 0,00 & 0,11 & 7,94 & 0,00 & 0,87 \\
\hline Psicología & 0,00 & 0,01 & 7,94 & 0,00 & 0,08 \\
\hline Coste & & & & 277,91 & 256,60 \\
\hline
\end{tabular}

Tabla 2. Uso, coste unitario y coste promedio por servicio en los dos modelos de atención. El uso y coste promedio se estimaron para un año. El coste se expresa en dólares estadounidenses.

presentó incremento, lo cual es francamente preocupante por tratarse de una población a la cual se le asignan periódicamente recursos para mantener o restituir la salud, convirtiéndose en una fuerte crítica al sistema de salud.

Los costes estimados en este trabajo se limitaron a los generados en el primer nivel de atención. Esto se podría considerar como una limitante ya que es una realidad que el paciente diabético en algún momento de la vida utilizará los servicios de segundo nivel, urgencias y de hospitalización, por ello hubiera sido conveniente incluirlos dentro de la estimación de los costos.

En un abordaje clínico epidemiológico el análisis inferencial determina qué alternativa es la mejor desde el punto de vista estadístico. No obstante, en un abordaje económico se podría cuestionar el uso de la estadística inferencial; por ejemplo, si no se encuentra diferencia estadísticamente significativa y la relación coste-efectividad identifica una de las dos alternativas, ¿se debe o no se debe adoptar para implementar en la práctica clínica? Y es aquí cuando pareciera que los diseños con abordaje epidemiológico y los diseños con abordaje económico toman distancia, y pareciera entonces que la alternativa más coste-efectiva, a pesar de no reportar significancia estadística, debería de ser la elección para llevar a la práctica.

Al comparar la efectividad, el grupo de la intervención educativa obtiene mejores resultados, pero la comparación del coste identifica a la estrategia médico familiar como la menos costosa. En el contexto de la economía de la salud $y$, específicamente en los estudios de coste-efectividad, la evaluación de la efectividad y del coste no se puede realizar de manera independiente. La evaluación se ejecuta en un sólo momento de manera conjunta. Para ello es útil la información presentada, la cual señala que por cada 100 \$US invertidos se desciende 10,42 mg/ dl de glucosa en sangre en la estrategia educativa y $2,57 \mathrm{mg} / \mathrm{dl}$ en la estrategia médico familiar. Esta comparación es válida y revela que entre las dos alternativas la mejor opción corresponde a la estrategia educativa.

No obstante, de manera paralela se debe cuestionar si invertir 100 \$US por paciente para descender 10,42 mg/dl corresponde a una 


\begin{tabular}{|c|c|c|c|c|c|}
\hline \multirow{2}{*}{ Coste de la intervención } & \multicolumn{2}{|c|}{ Estrategia Educativa } & \multicolumn{3}{|c|}{ Médico Familiar } \\
\hline & & 277,91 & & 256,60 & \\
\hline \multirow[t]{2}{*}{ Indicador } & $\begin{array}{c}\text { Unidades } \\
\text { ganadas }\end{array}$ & Coste & $\begin{array}{l}\text { Unidades } \\
\text { ganadas }\end{array}$ & & Coste \\
\hline & & \multicolumn{4}{|c|}{ Glucosa } \\
\hline Miligramos por decilitro & 28,96 & \multirow[b]{2}{*}{9,60} & \multirow{2}{*}{\multicolumn{3}{|c|}{38,88}} \\
\hline Coste por mg descendido & & & & & \\
\hline \multirow[t]{2}{*}{ Miligramos ganados por 100 \$US } & 10,42 & & & 2,57 & \\
\hline & & \multicolumn{4}{|c|}{$\underline{\text { Colesterol }}$} \\
\hline Miligramos por decilitro & 2,86 & \multirow[b]{2}{*}{97,17} & \multirow{2}{*}{\multicolumn{3}{|c|}{$8.553,49$}} \\
\hline Coste por mg descendido & & & & & \\
\hline \multirow[t]{2}{*}{ Miligramos ganados por 100 \$US } & 1,03 & & & 0,01 & \\
\hline & & \multicolumn{4}{|c|}{$\underline{\text { Triglicéridos }}$} \\
\hline Miligramos por decilitro & 13,73 & \multirow[b]{2}{*}{20,24} & \multirow{2}{*}{\multicolumn{3}{|c|}{256,60}} \\
\hline Coste por mg descendido & & & & & \\
\hline \multirow[t]{2}{*}{ Miligramos ganados por 100 \$US } & 4,94 & & & 0,39 & \\
\hline & & \multicolumn{4}{|c|}{$\underline{\mathrm{IMC}}$} \\
\hline $\mathrm{Kg} / \mathrm{m} 2$ & $-1,59$ & \multirow[b]{2}{*}{$-174,78$} & \multirow{2}{*}{\multicolumn{3}{|c|}{$-986,94$}} \\
\hline Coste por unidad descendida & & & & & \\
\hline Unidades ganadas por 100 \$US & $-0,57$ & & & $-0,10$ & \\
\hline
\end{tabular}

Tabla 3. Análisis coste-efectividad por estrategia educativa y médico familiar.

\begin{tabular}{ccc}
\hline \multirow{2}{*}{$\begin{array}{c}\text { Nivel de glucosa } \\
\text { de referencia }\end{array}$} & Estrategia Educativa & Médico Familiar \\
\cline { 2 - 3 } Inversión (\$US) & \multicolumn{2}{c}{187,97} \\
\hline 100 & Glucosa (mg/dl) & \\
200 & 150,82 & 185,40 \\
300 & 140,40 & 182,83 \\
400 & 129,98 & 180,25 \\
500 & 119,56 & 177,68 \\
600 & 109,14 & 175,11 \\
700 & 98,72 & 172,54 \\
800 & 88,29 & 169,97 \\
900 & 77,87 & 167,39 \\
1.000 & 67,45 & 164,82 \\
1.100 & 57,03 & 162,25 \\
1.200 & & 159,68 \\
1.300 & & 157,11 \\
1.400 & & 154,53 \\
\hline
\end{tabular}

Tabla 4. Inversión necesaria para alcanzar niveles de control de glucosa en estrategia educativa y médico familiar. 


\begin{tabular}{ccc}
\hline \multirow{2}{*}{$\begin{array}{c}\text { Nivel de colesterol } \\
\text { de referencia }\end{array}$} & Estrategia Educativa & Médico Familiar \\
\cline { 2 - 3 } & 196,63 & 197,93 \\
\hline Inversión (\$US) & \multicolumn{2}{c}{ Colesterol (mg/dl) } \\
\hline 100 & 196,63 & 197,92 \\
200 & 194,57 & 197,91 \\
300 & 193,54 & 197,89 \\
400 & 192,51 & 197,88 \\
500 & 191,48 & 197,87 \\
600 & 190,46 & 197,86 \\
700 & 189,43 & 197,85 \\
800 & 188,40 & 197,84 \\
900 & 187,37 & 197,82 \\
1.000 & 186,34 & 197,81 \\
\hline
\end{tabular}

Tabla 5. Inversión necesaria para alcanzar niveles de control de colesterol en estrategia educativa y médico familiar.

\begin{tabular}{ccc}
\hline \multirow{2}{*}{$\begin{array}{c}\text { Nivel de triglicéridos } \\
\text { de referencia }\end{array}$} & Estrategia Educativa & Médico Familiar \\
\cline { 2 - 3 } & 215,03 & 230,56 \\
\hline Inversión (\$US) & \multicolumn{2}{c}{ Triglicéridos (mg/dl) } \\
\hline 100 & 215,03 & 230,17 \\
200 & 205,15 & 229,78 \\
300 & 200,21 & 229,39 \\
400 & 195,27 & 229,00 \\
500 & 190,33 & 228,61 \\
600 & 185,39 & 228,22 \\
700 & 180,45 & 227,83 \\
800 & 175,51 & 227,44 \\
900 & 170,57 & 227,05 \\
1.000 & 165,62 & 226,66 \\
\hline
\end{tabular}

Tabla 6. Inversión necesaria para alcanzar niveles de control de trigliceridos en estrategia educativa y médico familiar.

asignación eficiente, pero ese análisis se encuentra fuera del alcance de este trabajo y queda para una futura investigación. Esta misma tendencia se aprecia cuando los indicadores empleados son el colesterol y los triglicéridos. Se dejó fuera del análisis el índice de masa corporal porque los resultados son contradictorios con la teoría, pero es una realidad de la población estudiada que se caracteriza por enfrentar una alta prevalencia de obesidad con tendencia al alza ${ }^{20,21}$.
Si bien es cierto muchos de los estudios de costeefectividad emplean datos secundarios producto de ensayos clínicos, también es una realidad que el uso de información procedente de otras poblaciones con características no necesariamente similares a la que se estudia puede llevar a resultados erróneos y, aún más, emplear una variedad amplia de fuentes de información puede ocasionar la pérdida de control. Es verdad que en este trabajo se obtuvo la información a partir de 
un estudio observacional. También es verdad que es información producto de la propia población y en consecuencia refleja su comportamiento, lo cual puede considerarse como una ventaja, más allá de que no corresponda a un ensayo clínico.

Cuando se compara la proyección del coste para alcanzar niveles de control de los indicadores bioquímicos, la estrategia educativa en todos los casos alcanza el control con una menor inversión económica, lo cual reafirma la tendencia ya descrita en el análisis coste-efectividad previo.

Con estos resultados no existe duda de que la elección de la estrategia de atención corresponde a la estrategia educativa, ya que es la que mejor relación coste-efectividad presenta. No obstante, se debe dejar en claro que la evaluación, y en consecuencia los resultados, corresponden a un análisis de corto-medio plazo y que en un escenario de largo plazo no necesariamente se obtendrán los mismos resultados.

En el contexto de la economía de la salud, que postula la asignación eficiente de los escasos recursos disponibles ${ }^{22,23}$, y más allá de los resultados aquí presentados, se debe cuestionar si disminuir la glucosa en sangre 10,42 mg/dl o 2,57 $\mathrm{mg} / \mathrm{dl}$ por cada 100 \$US invertidos corresponde a una asignación eficiente.

En conclusión, si bien es verdad se identifica la mejor alternativa coste-efectividad (estrategia educativa), se debe reconocer como realidad que ninguna de las dos opciones logra el control metabólico en la atención al paciente diabético, por lo cual es importante definir si asignar recursos en esta alternativa corresponde a una asignación eficiente y de no ser así, se deberán realizar modificaciones en ambas estrategias.

\section{BIBLIOGRAFÍA}

1. Garrido F, Lozano R, Tapia J, Martínez T, Rincón K, Ramírez A et al. Salud: México 2006. Información para rendición de cuentas. México, D.F. Secretaría de Salud. 2007; (55): 241-3. [acceso 10 de julio de 2012] Disponible en: http:// www.dged.salud.gob.mx/contenidos/dedss/descargas/ rcs/sm2006.pdf

2. Instituto Nacional de Estadística Geografía e Informática. Mujeres y hombres en México. INEGI. 2010 [acceso 8 de julio de 2012] Disponible en: http://www.inegi.org. $\mathrm{mx} /$ prod_serv/contenidos/espanol/bvinegi/productos/ integracion/sociodemografico/mujeresyhombres/2010/ MyH_2010.pdf

3. Gómez O, Sesma S, Becerril V, Knaul F, Arreola H, Frenk J. Sistema de salud en México. Salud Publica Mex. 2011; 53 (Supl 2): S220-S232.

4. Instituto Mexicano del Seguro Social. Guía de práctica clínica. Diagnóstico y tratamiento de la diabetes mellitus 2 en el primer nivel de atención. 2009: 11-24 [acceso 3 de julio de 2012] Disponible en: http://www.imss. gob.mx/profesionales/guiasclinicas/Documents/GER DiabetesMellitusTipo2.pdf

5. Garrido F, Tapia J, Ramírez A, Velázquez A, Cordero $M$, Vega $R$ et al. Rendición de cuentas en salud 2008.
Secretaría de Salud. 2009 [acceso 15 de julio de 2012] Disponible en: http://www.dged.salud.gob.mx/contenidos/ dedss/descargas/rcs/rcs2008.pdf

6. Instituto Mexicano del Seguro Social. Guía técnica para otorgar atención médica en el modulo de DiabetIMSS a derechohabientes con diagnóstico de diabetes mellitus, en unidades de medicina familiar. México 2008.

7. Secretaria de Salud. De atención médica. Artículo 24. Capítulo I, Prestación de los servicios de salud. Título tercero. Ley general de salud. México 2007. [acceso 15 de febrero de 2013] Disponible en: http://www.salud.gob.mx/ unidades/cdi/legis/lgs/index-t3.htm

8. Secretaría de Salud. Programa de acción: diabetes mellitus. México: Secretaria de Salud. 2001 [acceso 15 de febrero de 2013] Disponible en: http://www.salud.gob.mx/ unidades/cdi/documentos/diabetes_mellitus.pdf

9. Villarreal E, Vargas ER, Martínez L, Galicia L. El sistema de salud y el paciente con diabetes. México: UIESSQ-IMSS; 2005.

10. Bustos-Saldaña R, Bustos-Mora A, Bustos-Mora R, SolísRuiz ML, Chávez-Chávez MA, Aguilar-Núñez LM. Control de la glucemia en diabéticos tipo 2. Rev Med Inst Mex Seguro Soc. 2005; 43 (5): 393-9.

11. Gálvez A. Economía y salud en el camino hacia la eficiencia. Rev Cubana de Salud Pública. 2010; 36 (1):1-2.

12. Alba LH, Bastidas C, Vivas JM, Gil F. Prevalencia de control glucémico y factores relacionados en pacientes con diabetes mellitus tipo 2 del Hospital Universitario de San Ignacio, Bogotá, Colombia. Gac Med Mex. 2009; 145 (6): 469-74.

13. Blanco L, Villarreal E, Vargas E, Galicia L, Martínez L, Mejía A. Relación costo-efectividad de la cita previa en la consulta externa de medicina familiar. Aten Primaria. 2009; 41 (6): 329-34.

14. Escobar NV, Oliva A, Gracia Z. Aspectos bioéticos y socioeconómicos de la salud. Medisan. 1998; 2 (3): 36-44.

15. González-Pier E, Gutiérrez-Delgado C, Stevens G, BarrazaLloréns $\mathrm{M}$, Porras-Condey $\mathrm{M}$, Carvalho $\mathrm{N}$ et al. Definición de prioridades para las intervenciones de salud en el sistema de protección social en salud de México. Salud Publica Mex. 2007; 49 (Supl 1): S37-S52.

16. Aráuz AG, Sánchez G, Padilla G, Fernández M, Roselló M, Guzmán S. Intervención educativa comunitaria sobre la diabetes en el ámbito de la atención primaria. Rev Panam Salud Publica. 2001; 9 (3): 145-53.

17. López-Portillo A, Bautista-Vidal RC, Rosales-Velásquez OF, Galicia-Herrera L, Rivera y Escamilla JS. Control clínico posterior a sesiones grupales en pacientes con diabetes e hipertensión. Rev Med Inst Mex Seguro Soc. 2007; 45 (1): 29-36.

18. Oviedo MA, Espinosa F, Reyes H, Trejo JA, Gil E. Guía clínica para el diagnóstico y tratamiento de la diabetes mellitus tipo 2. Rev Med Inst Mex Seguro Soc. 2003; 41 (Supl): S27-S46.

19. American Diabetes Aassociation. Standards of medical care in diabetes 2012. Diabetes Care. 34 (1): 11-47.

20. Villa AR, Escobedo MH, Méndez-Sánchez N. Estimación y proyección de la prevalencia de obesidad en México a través de la mortalidad por enfermedades asociadas. Gac Med Mex. 2004; 140(Suplemento 2): S21-S25.

21. Braguinsky J. Prevalencia de obesidad en América Latina. An Sist Sanit Navar. 2002; 25 (Suppl 1): 109-15.

22. Thompson I. Definición de economía. Promonegocios.net [acceso 17 de febrero de 2013] Disponible en: http://www. promonegocios.net/economia/definicion-economia.html

23. Rodríguez-Ledesma MA, Vidal-Rodríguez C. Conceptos básicos de economía de la salud para el médico general. Rev Med Inst Mex Seguro Soc. 2007; 45 (5): 523-32. 